\title{
Competitive Proliferation of Aid Projects: A Model
}

By David Roodman

Abstract
The proliferation of aid projects may overburden recipient governments with
reporting requirements, donor visits, and other administrative overhead, siphoning
off scarce domestic recipient resources, such as tax revenue or the time of skilled
government officials, from directly productive use. But greater oversight may also
improve the administration of projects, increasing development. I present a model of
aid projects that reflects both sides of this coin. It posits a distinction between
national-level governance and project-level governance. A donor can raise project-
level governance above the baseline national level by requiring oversight activities
of the recipient, although the benefits from doing so are less where national-level
governance is already high. The model assumes that larger projects demand
proportionally less oversight activity from the recipient. Comparative statics analysis
suggests that to maximize development, projects should be larger where aid volume
is higher, to avoid overburdening recipient administrative capacity; where recipient
resources are scarcer, for the same reason; and where national governance is good,
since the marginal benefit of oversight is then lower. A multi-donor generalization
shows how donors that are imperfectly altruistic, caring most about the success of
their own projects, will tend to sink into competitive proliferation, in which each
donor subdivides its aid budget into smaller projects to raise the marginal
productivity of the recipient's resources in those projects and attract them away from
other donors. The inefficiency arises from the lack of a market among donors for
recipient resources. In a Nash equilibrium, competitive proliferation reduces overall
development. But the smallest (selfish) donors can gain. This would discourage them
from cooperating with other donors to contain competitive proliferation.

The Center for Global Development is an independent think tank that works to reduce global poverty and inequality through rigorous research and active engagement with the policy community. This Working Paper was made possible in part by funding from the William and Flora Hewlett Foundation.

Use and dissemination of this Working Paper is encouraged, however reproduced copies may not be used for commercial purposes. Further usage is permitted under the terms of the Creative Commons License. The views expressed in this paper are those of the author and should not be attributed to the directors or funders of the Center for Global Development.

www.cgdev.org 


\title{
Competitive Proliferation of Aid Projects: A Model
}

\author{
David Roodman ${ }^{1}$ \\ June 2006
}

\begin{abstract}
The proliferation of aid projects may overburden recipient governments with reporting requirements, donor visits, and other administrative overhead, siphoning off scarce domestic recipient resources, such as tax revenue or the time of skilled government officials, from directly productive use. But greater oversight may also improve the administration of projects, increasing development. I present a model of aid projects that reflects both sides of this coin. It posits a distinction between national-level governance and project-level governance. A donor can raise project-level governance above the baseline national level by requiring oversight activities of the recipient, although the benefits from doing so are less where national-level governance is already high. The model assumes that larger projects demand proportionally less oversight activity from the recipient. Comparative statics analysis suggests that to maximize development, projects should be larger where aid volume is higher, to avoid overburdening recipient administrative capacity; where recipient resources are scarcer, for the same reason; and where national governance is good, since the marginal benefit of oversight is then lower. A multi-donor generalization shows how donors that are imperfectly altruistic, caring most about the success of their own projects, will tend to sink into competitive proliferation, in which each donor subdivides its aid budget into smaller projects to raise the marginal productivity of the recipient's resources in those projects and attract them away from other donors. The inefficiency arises from the lack of a market among donors for recipient resources. In a Nash equilibrium, competitive proliferation reduces overall development. But the smallest (selfish) donors can gain. This would discourage them from cooperating with other donors to contain competitive proliferation.
\end{abstract}

\footnotetext{
${ }^{1}$ Research Fellow, Center for Global Development. droodman@cgdev.org. The author would like to thank his colleagues Peter Timmer, Steven Radelet, and Michael Clemens, as well as participants in a June 2005 seminar at the World Institute for Development Economics Research, for extremely useful comments.
} 


\section{Introduction}

The proliferation of projects is cited as one way in which the foreign aid delivery system is running amok (Morss 1984; van de Walle and Johnston 1996; Birdsall 2005). In her catalog of the “seven deadly sins” of aid delivery, Birdsall (2005) cites proliferation under “envy,” a heading that refers to the failure of donors to coordinate. Evidently, each donor wants its own school-building project, its own HIV prevention campaign, and so on.

In the old days, our elders tell us, higher moral standards prevailed in the donor community. According to Morss (1984), during the first two decades of foreign aid to developing countries, the 1950s and 1960s, most aid was given in the form of “program support.” By this he meant funding for large infrastructure projects or broad support for a sector such as agriculture or health, support that could include grants, loans, technical assistance, and commodities. However, in the 1970s, doubts about the effectiveness of aid, compounded by demands from legislatures for clear results, led to a shift toward project aid. Aid was committed and disbursed in smaller packets and goals were more limited and measurable—-say, the building of a certain number of schools. More than 20 years ago, Morss wrote that:

the proliferation of donor projects...is having a negative impact on the major government institutions of developing nations. Instead of working to establish comprehensive and consistent national development objectives and policies, government officials are forced to focus on pleasing donors by approving projects that mirror the current development "enthusiasm" of each donor. Further, efforts to implement the large number of discrete, donor-financed projects, each with its own specific objectives and reporting requirements, use up far more time and effort than is appropriate. Project consolidation is needed, but this is unlikely to occur on a significant scale because of the competitive nature of donor interactions.

Today, it is frequently claimed that Tanzania has to file 2,400 reports to donors every year and host 1,000 donor visits. Those particular numbers appear to be an urban legend started by a speechwriter for World Bank President James Wolfensohn, based on a misreading of van de 
Walle and Johnston (1996). ${ }^{2}$ But in the case of this urban legend, numbers everyone wants to believe are in fact reasonable, probably even conservative. Roodman (2006) counts more than 1,500 individual aid activity commitments to Tanzania during 2001-03—and that only puts the country in eighth place, behind Mozambique (with the most commitments), India, China, Russia, Ethiopia, Indonesia, and Vietnam. It is natural to wonder whether Ethiopia can efficiently oversee as many aid projects as Indonesia.

Roodman (2006) develops a microeconomic model of aid projects that focuses on the costs of proliferation. In that paper, all projects have the same technology, and take two inputsaid, and a recipient-side resource such as recurring expenditures or time of skilled officials. The total aid flow into a country and the distribution of that flow among projects of various sizes is taken as given. A notion of sunk cost is introduced, to represent the reports to be filed, the meetings to be attended, and the missions to be hosted, all of which divert recipient resources from direct use in aid-financed projects. Analysis shows that if the recipient is not a perfect development optimizer, but also cares about project “throughput” (which could be career benefits or kickbacks from association with operating projects) then under some circumstances increasing aid reduces development. In simulations, a threshold aid level appears beyond which the marginal benefit of an aid increase, given the proliferation that tends to come accompany it, is low or negative.

This paper starts by changing that model in one important way: the "sunk costs" of project oversight activities now come with a benefit. Oversight and accountability improve the quality of implementation of projects, or “project-level governance.” The marginal benefit,

\footnotetext{
${ }^{2}$ Van de Walle and Johnston (1996, p. 50) suggest that 600 simultaneous projects—about the number reported to be operating in Kenya and Zambia in the mid-1980s—would lead to 2,400 reports and more than 1,000 missions per year. They do so in a sentence that immediately follows one about Tanzania. But, in fact, the figure of 600 does not refer to Tanzania. They count more than 2,000 projects in Tanzania in the mid-1990s, implying even more reports and missions.
} 
however, declines in countries that are generally well-governed anyway. And the marginal cost rises where recipient administrative capacity is scarce. These trade-offs give rise to a notion of optimal project size at the recipient level. And it becomes possible to define optimal behavior on the part of the donor. In particular, it will be shown that projects should be larger in countries that a) are better governed, b) have less administrative capacity in absolute terms, or c) receive more aid—all else equal.

The paper then generalizes to multiple donors, showing how aid effectiveness can fall below the optimum because of proliferation of small projects. As in the pioneering work of Knack and Rahman (2004), the inefficiency occurs if donors are not perfectly altruistic, that is, if they care more about the success of their own projects than that of other donors' projects. "Competitive proliferation” then occurs, in which donors divide their aid budgets more finely in order to raise the marginal productivity of the recipient government's resources in those projects and divert the resources away from other donors’ projects. Since individual donors do not pay at the margin for the recipient inputs into the production processes they design, they experience the benefits of diversion but not the costs. Selfish donors thus generate a negative externality, which leads to a suboptimal equilibrium. Put otherwise, the development optimum - the set of projects from all donors that maximizes total development-is unstable. Theoretical analysis and simulations show that the smallest donors have the greatest incentive to proliferate and can even benefit from all-out competitive proliferation, even as larger donors and the recipient lose out. Donors could coordinate to prevent this outcome, but it would be difficult since the smallest donors would somehow need to be bought off.

\section{A microeconomic model of aid projects}

Knack and Rahman (2004) appear to be the first to devise a model relating to proliferation and test it empirically. The key moving part in their model is the skilled national, who can work 
either for a donor on a specific project, or, at lower pay, for the government, where he or she provides a public good that benefits all projects. Donors that hire nationals away from the government thus impose an externality on all other projects. The smaller a donor's share in a recipient's total aid flow, the more of the externality is imposed on other donors. Unless a donor is perfectly altruistic, the smaller it is, the greater its tendency to deviate from the optimum by proliferating projects and hiring skilled nationals away from the government. The empirical prediction is that countries receiving aid flows more fragmented among donors, as measured by a Herfindahl-Hirschman index, will have lower bureaucratic quality in government. Knack and Rahman find that the data uphold the prediction.

The model described here is similar in spirit to that of Knack and Rahman while departing from it in important ways. In particular, it generates norms or predictions about how donors size projects. The aid process is a set of production activities (projects), with identical technologies. A project, indexed by $i$, has two inputs: aid, $A_{i}$, and a recipient-side resource, $R_{i}$. I will refer to $A_{i}$ as the project's “size.” The recipient resource can be thought of as the time of skilled government bureaucrats, as in Knack and Rahman, or funds for recurring costs, for example. The resource is subject to a fixed national-level budget constraint. Each project has a monitoring cost that the recipient must cover out of that general budget in order for the project to go forward. The monitoring cost is assumed to rise slower than project size, with constant elasticity:

$$
\begin{aligned}
& s\left(A_{i}\right)=s_{1} \cdot\left(A_{i}\right)^{c} \\
& \text { where } s_{1}>0 \text { and } 0 \leq c<1 .
\end{aligned}
$$

The coefficient $s_{1}$ is not unitless; rather, it converts between the units for $A_{i}$, presumably aid dollars, and those of $R_{i}$, which could be dollars or local currency or number of people. Having noted this subtlety, I will henceforth assume that units are such that $s_{1}=1$, and drop it. 
The “development" produced by a project, $D_{i}$, is the product of two non-negative factors. The first, called "output” or $O_{i}$, can be thought of as the result of a mechanical process, described by a standard production function, that combines the donor's aid and the recipient's resource input, the latter being net of what the recipient must spend on monitoring activities. The second factor in development is project-level governance, $G_{i}$, which is a function of the recipient's general level of governance, $G$, and the “monitoring cost ratio," the ratio of monitoring cost to project size. $G_{i}$ is positive except in the theoretical limit where national-level governance, $G$, is 0 and there is no monitoring. The idea is that the recipient's overall governance quality is a major determinant of project success, but by requiring enough monitoring and oversight, the donor can arbitrarily raise the effective level of governance for a given project. Thus:

$$
\begin{aligned}
& D_{i}=O_{i} G_{i} \\
& O_{i}=o\left(A_{i}, R_{i}-s\left(A_{i}\right)\right) \\
& G_{i}=g\left(s\left(A_{i}\right) / A_{i}, G\right) . \\
& G_{i}=0 \Rightarrow s\left(A_{i}\right)=G=0
\end{aligned}
$$

This governance factor is the major innovation over Roodman (2006). By associating benefits as well as costs with the monitoring effort of reports and meetings, analysis of project proliferation becomes a matter not just of pure cost, but trade-offs.

Implicit in this construct is a signaling or principal-agent problem between donor and recipient. The donor cannot observe, with perfection and without cost, the recipient's participation in and management of projects. It therefore makes its aid disbursement conditional on the recipient performing certain easily observed project-monitoring activities and on satisfactory evaluation results flowing from those activities. This creates an incentive for the recipient to participate in a way that raises project efficiency, but of course comes at a cost in recipient resources. 
This leads to a paradox. We will assume throughout for the sake of tractability that the recipient is a development maximizer. Why then need the donor monitor at all? The way out of the paradox is to imagine a central authority on the recipient side, perhaps a ministry of finance, that can allocate resources among projects but has incomplete control over the quality of management, technical skill level, corruption, and other factors within the line ministries that use the resources. The allocator has the propensity to perfectly maximize development within the ambit of its powers, while the line ministries generally do not. Like all such assumptions, this one is simplistic but makes for a tractable model that focuses on dynamics of interest.

Total development from a set of $N$ projects is

$$
D=\sum_{i} D_{i}
$$

In other words, we assume that projects do not interact at either the microeconomic level. Nor do they at the macroeconomic level, as they might in a general equilibrium framework; in other words, they are implicitly assumed to represent a modest share of GDP.

We also assume that $o$ is homogeneous of degree $d$, so that it has declining, constant, or increasing returns to scale according to whether $d$ is less than, equal to, or greater than 1 . Since a project will only go forward if the recipient invests enough in a project to more than cover the monitoring cost,

$$
o\left(A_{i}, R_{i}-s\left(A_{i}\right)\right)=0 \text { if } R_{i}-s\left(A_{i}\right)<0 .
$$

This condition can make $o$ discontinuous at the point where monitoring costs are just covered.

For example, if $o\left(A_{i}, R_{i}-s\left(A_{i}\right)\right)=A_{i}+R_{i}-s\left(A_{i}\right)$, output will equal 0 if the recipient falls a penny short of covering monitoring costs, but jump to $A_{i}$ if the recipient puts in that last penny. (But if $o$ is Cobb-Douglas, it is continuous at $R_{i}-s\left(A_{i}\right)=0$ since Cobb-Douglas production is zero when any of its inputs is.)

Otherwise, we assume that $o$ and $g$ are twice differentiable. We impose first- and secondorder assumptions: 


$$
\begin{gathered}
o_{1}, o_{2}>0 ; o_{11}, o_{22}<0 ; o_{12}>0 \\
g_{1}, g_{2}>0 ; g_{11}, g_{12} \leq 0
\end{gathered}
$$

where the subscripts denote differentiation with respect to first or second arguments. ${ }^{3}$ In words, output is positive in either factor alone, but with decreasing marginal returns. In the $o$ production process, increasing either factor raises the marginal product of the other. As for project-level governance, it rises with the ratio of monitoring cost to project size, and with the recipient's national-level governance, $G$. As either factor increases, the marginal responsiveness of projectlevel governance to further monitoring falls, as would be the case if there were an asymptotic limit to $g$ ("perfect governance”). ${ }^{4}$ From these conditions arise the basic problem donors have apparently struggled with for decades: larger projects, including what are called programs, impose less overhead cost on recipients since monitoring costs rise slower than project size. But less monitoring can reduce the project’s “governance,” the more so in countries that are generally poorly governed.

The next three sections state and analyze three problems based on this model. In the first, there is one donor, who chooses both how much aid to give and in how many projects, in order to maximize development. The section shows that sometimes there is an optimal aid volume and an optimal project size. The second differs in taking total aid as exogenously determined, which the literature demonstrates that it is a substantial extent (discussed below), leaving only project size as a parameter to be chosen by optimization. The third introduces multiple donors. The analysis

\footnotetext{
${ }^{3}$ Strict inequalities are mostly used here to avoid extreme cases such as Leontif production that complicate analysis and exposition.

${ }^{4} \mathrm{~A}$ function that satisfies these conditions is

$$
G_{i}=1-e^{-\gamma \frac{s\left(A_{i}\right)}{A_{i}}}(1-G)=1-e^{-\gamma A_{i}^{c-1}}(1-G) \text {, where } \gamma>0 .
$$

Here, as the sunk-cost ratio rises from 0 , project-level governance rises asymptotically from the baseline of $G$ to 1 . $G=1$ can be thought of as perfect national-level governance; if $G=1$, then $G_{i} \equiv 1$, and monitoring has no benefit.
} 
here is not exhaustive; to limit complexity, it fully investigates interiors solutions only. But it goes far enough to generate plausible norms for donor behavior.

\section{An optimal aid level?}

If donor and recipient are jointly optimizing development, what is the optimal suite of projects to fund? In this section, we analyze that problem taking the recipient resource budget, $R$, and the national governance level, $G$, as given but letting the total aid budget, $A$, be a free parameter for optimization. Available aid is unlimited.

In general, by symmetry, it is reasonable to assume that all projects in the optimal distribution are the same size. So the problem for this section can be cast as, "what is the optimal size and number of (identical) projects?” The question is easiest to answer when treating the number of projects, $N$, as continuous. At the optimum, the recipient's budget constraint will bind, and each project will receive

$$
\begin{aligned}
& \bar{A} \equiv \frac{A}{N} \text { in aid and } \\
& \bar{R} \equiv \frac{R}{N} \text { in recipient resource. }
\end{aligned}
$$

Total development is then, by (1), (2), and (3),

$$
D=\sum_{i} D_{i}=N \cdot o(\bar{A}, \bar{R}-s(\bar{A})) \cdot g(s(\bar{A}) / \bar{A}, G)=N \cdot o\left(\bar{A}, \bar{R}-\bar{A}^{c}\right) \cdot g\left(\bar{A}^{c-1}, G\right)
$$

Donor and recipient jointly solve

$$
\begin{aligned}
& \max _{A, N} D \\
& \text { given } R, G .
\end{aligned}
$$

That this maximum could exist at all is perhaps counterintuitive, since the freedom to increase one input, aid, without limit should seemingly open up vast production possibilities. But a marginal aid increase must expand either the project count or the project size (or both), and both possibilities have costs. The cost of increasing the number of projects while holding their size 
constant is that the recipient must devote more of its resources to covering monitoring costs, leaving less for direct production. The cost of increasing project size (while holding project count constant) is that monitoring efforts do not rise as fast, reducing the quality of governance for the projects.

Whether a maximum exists depends in part on how low national governance is. For a minimally arbitrary example, set $G$ to 0.5 (notionally middle-of-the-road), $c=0.5$ (similarly), and $R=100$. Let $o$ take the Cobb-Douglas form, $o(\bar{A}, \bar{R}-s(\bar{A}))=\bar{A}^{0.5}(\bar{R}-s(\bar{A}))^{0.5}$. And give $g$ the form in footnote 4, which is designed so that governance rises asymptotically toward 1 as the monitoring cost ratio rises: $g\left(s\left(A_{i}\right) / A_{i}, G\right)=1-(1-G) e^{-s\left(A_{i}\right) / A_{i}}$. Here, software can confirm, $D$ can indeed be increased without limit by expanding $A$ and bringing $N$ arbitrarily close to zero. In this case, the donor would fund a small fraction of a very large project; it would mandate essentially no recipient monitoring and effort, but would get positive output thanks the ambient quality of governance. However, if $G$ goes lower, to 0.1 , optimization software reports that development hits a maximum when $A=50.92$ and $N=49.03$, so that each project receives 1.038 units of aid and 2.039 units of recipient resource; exactly half of the latter (1.019) goes to sunk costs. Low-enough governance constrains aid absorption.

We use star superscripts to signify the values of variables at the solution to (7). Of particular interest is how $A^{*}$ and $N^{*}$, when they exist, vary with the parameters $R$ and $G$. As the numerical example suggests, and as Appendix 1 demonstrates, increasing national-level governance always increases the optimal project size and the optimal amount of aid-the recipient is more "aidworthy." Similarly, a rise in the recipient resource budget also expands the recipient's ability to absorb aid. In fact, it does so in direct proportion, so that the optimal project 
size remains unchanged. That is, $e_{R}^{A^{*}}=e_{R}^{N^{*}}=1$, where the notation $e_{Y}^{X}$ indicates the total elasticity of $X$ with respect to $Y$.

In other words, expanding the recipient's resource budget simply multiplies the number of projects implemented, each with the same donor and recipient resources as before. So total development also rises proportionally: $e_{R}^{D^{*}}=1$. And by the Envelope Theorem, the total elasticity of $D^{*}$ with respect to $R$ is the same as the partial elasticity (analogous to the partial derivative) of $D$ with respect to $R: e_{R}^{D^{*}}=\varepsilon_{R}^{D}$. This has an interesting consequence if $o$ is CobbDouglas as in the earlier numerical example, taking the form $o(\bar{A}, \bar{R}-s(\bar{A}))=\bar{A}^{\alpha}(\bar{R}-s(\bar{A}))^{\beta}$. In this case, by the chain rule for elasticities, $1=\varepsilon_{R}^{D}=\varepsilon_{2}^{o} \varepsilon_{R}^{2}$, where $\varepsilon_{2}^{o}$ represents the elasticity of $o$ with respect to its second argument, and $\varepsilon_{R}^{2}$ is the elasticity of that argument, $\bar{R}-\bar{A}^{c}$, with respect to $R$. It can be checked that $\varepsilon_{R}^{2}=\frac{\bar{R}}{\bar{R}-\bar{A}^{c}}$. And $\varepsilon_{2}^{o}=\beta$. Putting all this together gives

$$
\begin{gathered}
1=\beta \frac{\bar{R}}{\bar{R}-\bar{A}^{c}}, \\
\text { whence } \frac{\bar{R}-\bar{A}^{c}}{\bar{R}}=\beta .
\end{gathered}
$$

This last fraction is the portion of the recipient's resource not consumed by monitoring costs. In the numerical example, $\beta=0.5$, which is why there, sunk costs consume exactly half the recipient's resources.

\section{Fixed aid budget: single-donor case}

The previous section endogenized aid. This led to the sensible predictions that bigger, bettergoverned countries get more aid, but the assumption that aid is purely endogenous is unrealistic since aid has been demonstrated to have a substantial exogenous component. The well-known small-country bias, for example, sends more aid per person or unit of GDP to smaller countries 
(Mosley 1981; Alessina and Dollar 1998; Collier and Dollar 2002). Countries’ endowments of oil and other natural resources and their perceived importance in such overarching security strategies as the Cold War and the "Global War on Terror," also figure prominently (Kaplan 1975; McKinley and Little 1979; Schraeder, Hook, Taylor 1998; Moss, Roodman, and Standley 2005). The next two sections therefore take total aid, $A$, as given. This simplifies the mathematics by turning a two-dimensional optimization problem into a one-dimensional one. And it leads to somewhat different predictions.

The problem is now:

$$
\begin{aligned}
& \max _{N} D \\
& \text { given } A, R, G .
\end{aligned}
$$

A complete analysis requires examining boundary solutions. But since the purpose of this exercise is to motivate plausible and testable hypotheses, the details of such an analysis are omitted. Briefly, there are two extrema one could consider. One is the $N \rightarrow 0$ limit. Since $N$ is a continuous variable, one must imagine that as $N \rightarrow 0$, a mere fraction of a very large project is being implemented. If economies of scale are great enough (if $d$ is high enough), and nationallevel governance is above zero, so that project-level governance stays above zero even in the absence of monitoring, this "budget support extreme” can in fact be optimal.

The other extreme is the point, call it $\hat{N}$, at which sunk costs consume the recipient's entire resource budget. At this "project oversight extreme," the recipient devotes itself solely to supplying monitoring information to the donor and puts no resources into project implementation — no domestic covering of recurring costs, no domestic project implementation staff, etc. This extreme is most likely to be optimal if $d$ is small, meaning that strong diseconomies of scale favor small projects (with higher monitoring demands); if $c$, the elasticity of sunk cost with respect to project size, is small, so that sunk costs do not rise too fast as 
projects shrink; if $o_{2}$ is small at $\hat{N}$, that is, if the opportunity cost of recipient resources for direct production is low; if $\varepsilon_{1}^{g}$, which embodies the marginal governance benefit of monitoring, is high at $\hat{N}$; and if $R$ is low relative to $A$, which is to say, recipient resources are scarce.

Mathematically, $\hat{N}$, is defined by $\bar{R}=s(\bar{A})$, i.e., $R / \hat{N}=(A / \hat{N})^{c}$, the solution to which is

$$
\hat{N}=A^{-\frac{c}{1-c}} R^{\frac{1}{1-c}} \text {. }
$$

And at $\hat{N}$, the average project size is

$$
\overline{\hat{A}}=\frac{A}{\hat{N}}=\left(\frac{A}{R}\right)^{\frac{1}{1-c}} .
$$

If the project oversight extreme is an optimum in some region of parameter space, what does (10) say about the dependence of the optimal project size on the parameters? At the margin, average project size should rise with total aid, fall with recipient resource endowment—with equal and opposite elasticity—and not depend on national governance level. However, a nonmarginal increase in national-level governance could dislodge the optimum from the project oversight extreme. It would increase project-level governance, reduce the marginal benefit of monitoring, and could make a project count lower than $\hat{N}$, and a project size greater than $\overline{\hat{A}}$, optimal.

Another complexity is that if the optimal aid level is finite and the donor exceeds it, then a development-maximizing recipient will withhold its resources from some projects, forcing them to shut down for lack of monitoring, and devote its resources to a subset that collectively receive the optimal aid amount. ${ }^{5}$ In this case, the optimum will depend at the margin on the parameters $G$ and $R$ as described in the previous section, but not on $A$. Gross downward changes in $A$,

\footnotetext{
${ }^{5}$ This recipient behavior of defunding projects arises for somewhat different reasons in Roodman (2006).
} 
however, all else equal, could break the system out of this mode, shifting the optimum to an interior solution.

Indeed, in the remainder of this section, we analyze a classic interior solution to (7), where

$$
\begin{aligned}
& \frac{\partial D}{\partial N}=0 \\
& \frac{\partial^{2} D}{\partial N^{2}}<0
\end{aligned}
$$

In general, it is impossible to solve the first-order condition analytically for the optimum, $N^{*}$. But as in the previous section, we can investigate the behavior of $N^{*}$ with respect to marginal changes in the parameters, which we do in Appendix 2. The findings are three:

Better national-level governance favors fewer projects, thus, holding total aid constant, larger projects. Higher national-level governance reduces the scope for improving project-level governance through project oversight. Since the marginal benefit of project oversight is lower, the optimum shifts in favor of fewer, larger projects, for which the recipient is required to devote relatively less effort to oversight activities.

The larger the recipient's budget, the higher the optimal project count, and the smaller the optimal project size, all else equal. It may seem counterintuitive that recipients with larger budgets should have smaller projects. But countries with more government resources have fewer administrative bottlenecks and can absorb smaller, more numerous projects with all the attendant meetings and reports. The precise economic story is a bit tricky and runs as follows. If $R$ increases, the amount of recipient resource directly used in production in each project, rather than monitoring, increases. Because there are diminishing returns to this resource in production, the marginal opportunity cost of instead consuming the resource in monitoring then goes down. Because the marginal cost of monitoring has gone down, when donor and recipient then re-solve the optimization problem, they will shift slightly toward smaller projects with relatively higher 
monitoring costs. There is an other effect in the opposite direction, but it is smaller: increasing $R$ raises the marginal productivity of the complementary resource in $o$ production, namely aid, which in itself favors a tilt toward more aid per project and fewer projects.

If aid to a country rises, the optimal project count grows more slowly or even shrinks, so the optimal project size increases. The microeconomic story here is that increasing $A$ affects $\partial D / \partial N$, the marginal development gain from moving to more numerous, smaller projects, in three ways. First, the shift to larger projects reduces the monitoring cost ratio. Since there are diminishing returns to monitoring in terms of project-level governance, curtailing monitoring increases the marginal benefit of a shift (back) to smaller, more-monitored projects. So to this extent, a higher $A$ raises the marginal benefit of shifting to more, smaller projects. Second, because of diminishing returns in the $o$ production process, increasing aid per project reduces the marginal product of that aid. That reduces the opportunity cost of bringing aid per project back down by splitting the aid budget into smaller, more numerous projects. So here too more aid favors more projects. On the other hand, and third, increasing aid per project improves the marginal productivity of the recipient's complementary resource in o production, which raises the opportunity cost of consuming it with monitoring. This reduces the gain from moving to more, smaller projects, which involve more monitoring. Whether the positive or negative effects dominate depends on the details of the production technology and the parameters. But the balance of the effects is such that $N^{*}$ will never grow as fast as $A$. As result the optimal project size always increases with aid.

In sum, if donor and recipient jointly maximize development, and if $A$ is exogenously determined and does not exceed the optimal aid level (if it exists), donors should fund larger projects or programs in countries that are better governed, out of trust); in countries with limited 
government budgets, to avoid overload; or in countries with large aid flows, for the same reason.

The possibility of a budget support optimum where governance is high enough is consistent with this message. The positive relationship between governance and project size echoes a result from the endogenous-aid set-up in the previous section. The negative relationship with the recipient budget, however, is new. One potentially counterintuitive result is that, assuming the recipient's budget is proportional to gross domestic product, all else equal, larger countries should have smaller projects.

\section{Fixed aid budgets: multiple-donor case}

The purpose here, in the spirit of Knack and Rahman (2004), is to show that if there are multiple donors, and they each care most about the success of their own projects, then a negative externality will arise through competition for scarce recipient resources. This creates an incentive for “competitive proliferation” and leaves the recipient worse off. Partial analysis will also suggest that smaller donors generate a proportionally larger externality and so tend to proliferate more, meaning that they fund smaller projects. After describing the multi-donor framework in general terms, we specialize to the case of two partially selfish donors. The recipient is still assumed to maximize development in the allocation of its own resource.

In the new framework, there are $J$ donors, indexed by $j$. Each has an aid budget, $A_{j}$, that is still exogenous. Again by symmetry, all of the projects of a given donor are assumed to have the same size, so that project distributions can be characterized by project counts, $N_{j}$. Adapting the set-up from the single-donor case,

$$
\begin{aligned}
& D_{j}=N_{j} \cdot o\left(\bar{A}_{j}, \bar{R}_{j}-s\left(\bar{A}_{j}\right)\right) \cdot g\left(s\left(\bar{A}_{j}\right) / \bar{A}_{j}, G\right) \\
& D=\sum D_{j} .
\end{aligned}
$$

There are now $J+1$ actors, counting the recipient. The recipient's problem is: 


$$
\begin{aligned}
& \max _{\left(R_{j}\right)} D \\
& \text { such that } \sum R_{j} \leq R \\
& \text { given } R, G,\left(A_{j}\right),\left(N_{j}\right) .
\end{aligned}
$$

Let $0 \leq \alpha_{j} \leq 1$ be a donor-specific “altruism” parameter as in Knack and Rahman (2004) that determines how much weight donor $j$ gives to the success of other donors' projects relative to its own. Donor $j$ 's objective function is

$$
D_{j}+\alpha_{j} \sum_{k \neq j} D_{k}
$$

The situation, then, can be viewed as a game. The recipient's strategy has to do with its budget allocation across different donors' projects. Each donor's strategy is the choice of one variable, $N_{j}$ : its sole decision is how finely to divide its aid budget into projects.

It is important to appreciate the essential difference between this set-up and classic model of competing private actors, in which competition can only do good. It is that there is no market for the recipient's resources. The donors do not pay for the resources the way private firms pay for their inputs. As result, even though the optimizing recipient does allocate its resources so as to equate marginal products across donors the way market competition would, if a donor succeeds in attracting recipient resources away from another donor, it experiences the benefit but not the cost. We will explore how this externality can lead to inefficiency.

If the situation is treated as a game with $J+1$ players, including the recipient, then the development optimum described in the previous section translates into a multiplayer optimum that is a Nash equilibrium. This optimum consists of a total of $N$ identical projects, with $\bar{A}_{j}$ and $\bar{R}_{j}$ the same for all donors. This point, which we will call the "development optimum” since it does maximize total development, is clearly an optimum from the point of view of the development-maximizing recipient. And at this point, each donor's problem, holding constant 
the choices of the other donors and, importantly, the recipient, is a microcosm of the global optimization problem in the previous section, because all donors' projects are the same universal size and have the same recipient resource input per project. If a single donor can increase development from its projects by subdividing them more or less finely (holding the recipient's resource allocation across donors fixed), then all donors can do so, and the development optimum is not an optimum after all. Since no player can unilaterally improve its objective function, the development optimum is indeed a Nash equilibrium.

The game becomes more interesting if each donor is assumed to anticipate the recipient's response to the donor's choice of $N_{j}$. The recipient then disappears as a player, taking on a role analogous to that of the consumer in analysis of oligopoly, the vehicle of interaction among the producers. Then, a donor's choices can attract recipient resources away from other donors.

The remainder of this section partially analyzes that game. For simplicity, it focuses again on classic interior solutions. Let the vector $\left(R_{j}^{*}\right)$ be the recipient's solution to (13), and $\left(D_{j}^{*}\right)$ be total development from each donor's set of projects at this solution. Donor $j$ 's problem is:

$$
\begin{aligned}
& \max _{N_{j}} D_{j}^{*}+\alpha_{j} \sum_{k \neq j} D_{k}^{*} \\
& \text { given } G,\left(A_{k}\right),\left(N_{k}\right)_{k \neq j} .
\end{aligned}
$$

For simplicity now, we will assume there are just two donors.

Broadly speaking, each donor can pursue one of two strategies. It can cooperate, selflessly sizing its projects in accordance with the development optimum. Or it can compete, as Appendix 3 spells out, adjusting its project count to attract the recipient's resources away from the other donor's projects, thereby raising development from its own projects while reducing total development (since it moves away from the development optimum). There are thus three 
kinds of possible outcomes in the two-donor game: the donors cooperate; one "cooperates” while the other competes; and both compete. Appendix 3 shows that for a donor to lure the recipient's resources away from the other donor, the direction in which it must adjust its project count is upward. It must proliferate, subdividing its aid budget more finely than is optimal for the recipient overall. If the donor is sufficiently selfish, it will gain from such a unilateral move. Of course, the other donor may respond in kind.

Immediately, then, we can fill in three of the four boxes in the matrix for this game. If both donors cooperate, all projects will be the same size, and the development optimum will be achieved. But unless donors are perfectly altruistic, this outcome is not stable. If one donor cooperates while the other instead competes, the competing one will gain at the expense of the cooperating one, and at the expense of the recipient. This too tends to be unstable, since if the cooperating donor is actually selfish enough, it too can gain by unilaterally switching to the compete strategy.

Less clear is exactly what happens if both donors choose the compete strategy. In general, a Nash equilibrium in pure strategies does exist in this case; it is the solution to the system,

$$
\begin{aligned}
& \frac{d D_{1}^{*}}{d N_{1}}+\alpha_{1} \frac{d D_{2}^{*}}{d N_{1}}=0 \text { (maximization of donor 1's utility) } \\
& \frac{d D_{2}^{*}}{d N_{2}}+\alpha_{2} \frac{d D_{1}^{*}}{d N_{2}}=0 \text { (maximization of donor 2's utility) } \\
& \frac{\partial D_{1}}{\partial R_{1}}=\frac{\partial D_{2}}{\partial R_{2}} \text { (maximization of recipient's utility) } \\
& R_{1}+R_{2}=R \text { (recipient budget constraint), }
\end{aligned}
$$

along with appropriate second-order conditions. Two important questions arise about such an equilibrium: Which donors tend to proliferate most, small ones or large ones? And might one donor do better if both compete than if both cooperate? If some donors actually gain from 
universal competitive proliferation, then it will be harder for donors to agree as a group not to compete- to coordinate. Full answers to these questions are elusive because of the generality of the specification of $o$ and $g$ and the complexity of the above system of equations. Moreover, by (32) and (33) in Appendix 3, the total derivatives in the first two equations are functions of second partial derivatives of $D$, so the derivative of solutions to the system with respect to the parameters involve third derivates. Understanding the effect of non-marginal changes in parameters is even harder.

Here we will offer two pieces of an analysis that give some insight, one theoretical, one computational. At the theoretical level, Appendix 3 demonstrates that right at the development optimum, large, perfectly selfish donors feel less incentive to proliferate-to depart from that optimum - than small, perfectly selfish ones, in the sense that $e_{N_{j}}^{D_{j}^{*}}$, the elasticity of development from their projects to the project count, factoring in recipient response, is lower for big donors. The result is intuitive. As Knack and Rahman (2004) suggest, larger donors have relatively less incentive to lure recipient resources away from other donors. They internalize more of the externality of proliferation. But this conclusion technically only holds right at the development optimum, so it does not describe how much donors will actually proliferate before they reach a fully competitive equilibrium.

For more insight, we turn to simulations. We adapt the numerical example from section 0, so that $R=100, G=0.1, o(\bar{A}, \bar{R}-s(\bar{A}))=\bar{A}^{0.5}(\bar{R}-s(\bar{A}))^{0.5}, s\left(A_{i}\right)=\left(A_{i}\right)^{0.5}$, and $g\left(s\left(A_{i}\right) / A_{i}, G\right)=1-e^{-s\left(A_{i}\right) / A_{i}}$. This time, we assume there are two donors, that they are equally altruistic $\left(\alpha_{1}=\alpha_{2}\right)$, and that both donors choose to compete. In the first simulation, Donor 1 deploys 20 units of aid while donor 2 deploys 1 . Figure 1 shows how key variables evolve as 
altruism declines from 1 to 0 . At the left side, where $\alpha_{1}=\alpha_{2}=1$, the donors jointly achieve the development optimum. Their projects are identical $\left(\bar{A}_{1}=\bar{A}_{2}, \bar{R}_{1}=\bar{R}_{2}\right)$, differing only in number, and as a result $R_{1}$ and $R_{2}, N_{1}$ and $N_{2}$, are in the same 20:1 ratio as $A_{1}$ and $A_{2}$ - they are the same distance apart on the log scale. As altruism declines, however, the small donor's project count $\left(N_{2}\right)$ escalates rapidly, while the large donor's projects multiply more slowly, confirming smaller donors' tendency to proliferate more. At the right extreme, where altruism evaporates, the small donor's project count has climbed from 3.3 to 256, while the large one’s has gone from 66 to 82. The larger donor experiences a steady deterioration in the output of development from its projects, from 29.3 to 25.2 . The small donor actually gains as altruism falls-at the margin until altruism goes below 0.8, and overall through to the very end. Output from its projects starts at 1.47, rises to 1.88 , then slides to 1.62, as the war of proliferation takes a toll at the margin even on the small donor. The recipient shifts from putting $4.8 \%$ of its resources into the small donor's projects to $18.6 \%$ even though the small donor has not increased its aid giving. Total development from all projects falls from 30.8 to 26.8. In sum, the smaller donor benefits from competitive proliferation, but at the expense of a massive increase in project count, a diversion of recipient resources, and a 13\% decline in development. 
Figure 1. Dependence of key variables on altruism in Nash equilibria, Cobb-Douglas production, $A_{1}=20, A_{2}=1$

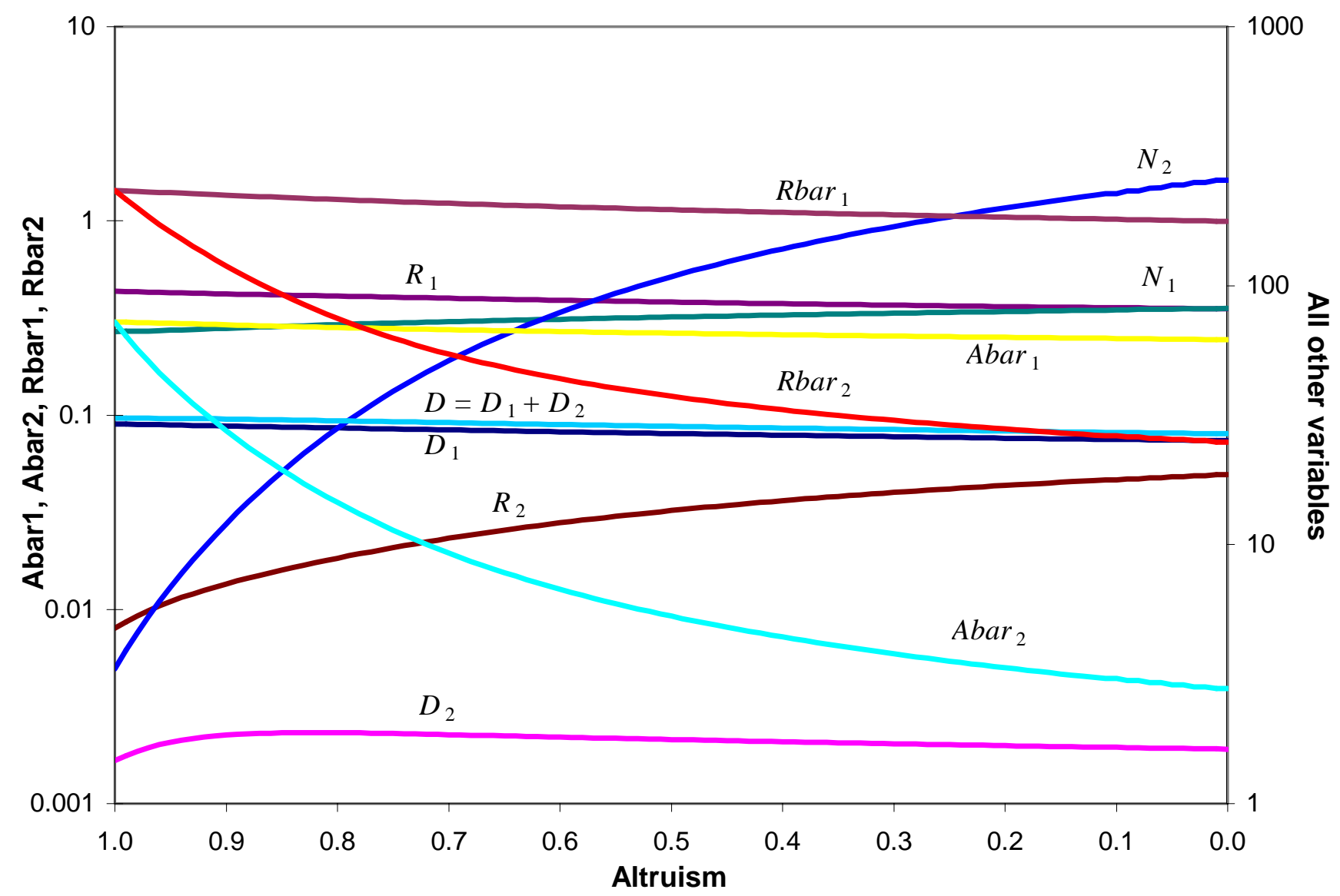

Adjusting the parameters does not change the story radically, but the incremental changes are interesting. As $A_{1}$ rises toward $A_{2}$, there comes a point where even the small donor loses under unbridled competitive proliferation. This must be so, for if $A_{1}=A_{2}$, by symmetry, the two donors will share equally in the (diminished) development pie.

Meanwhile, increasing the elasticity of substitution between aid and the recipient resource (via a symmetric, constant-returns-to-scale CES production function for $o$ ) inhibits proliferation—but increases the aggregate cost for development of what proliferation does occur. Why? What restrains the donors from proliferating ad infinitum is the cost of doing so for their 
own projects, namely the diversion of the recipient resource from productive use in the $o$ production process to the increased monitoring of smaller projects. And if the elasticity of substitution between aid and the recipient resource is high, then the limited availability of aid to a (very small) project is less of a constraint on the marginal productivity of the recipient's resource in direct production. The marginal productivity of the recipient's resource being higher, so is the opportunity cost of consuming it with monitoring. This puts a firmer limit on the extent of proliferation, but also leads to greater costs overall in the equilibrium. Figure 2 shows how the simulation changes when the elasticity of substitution switches from 1 , the Cobb-Douglas case, to 2 . The small donor's projects multiply by a factor of 66 instead of 77 , but total development falls $15 \%$ instead of $13 \%$. 
Figure 2. Dependence of key variables on altruism in Nash equilibria, CES production, elasticity of substitution=2, $A_{1}=20, A_{2}=1$

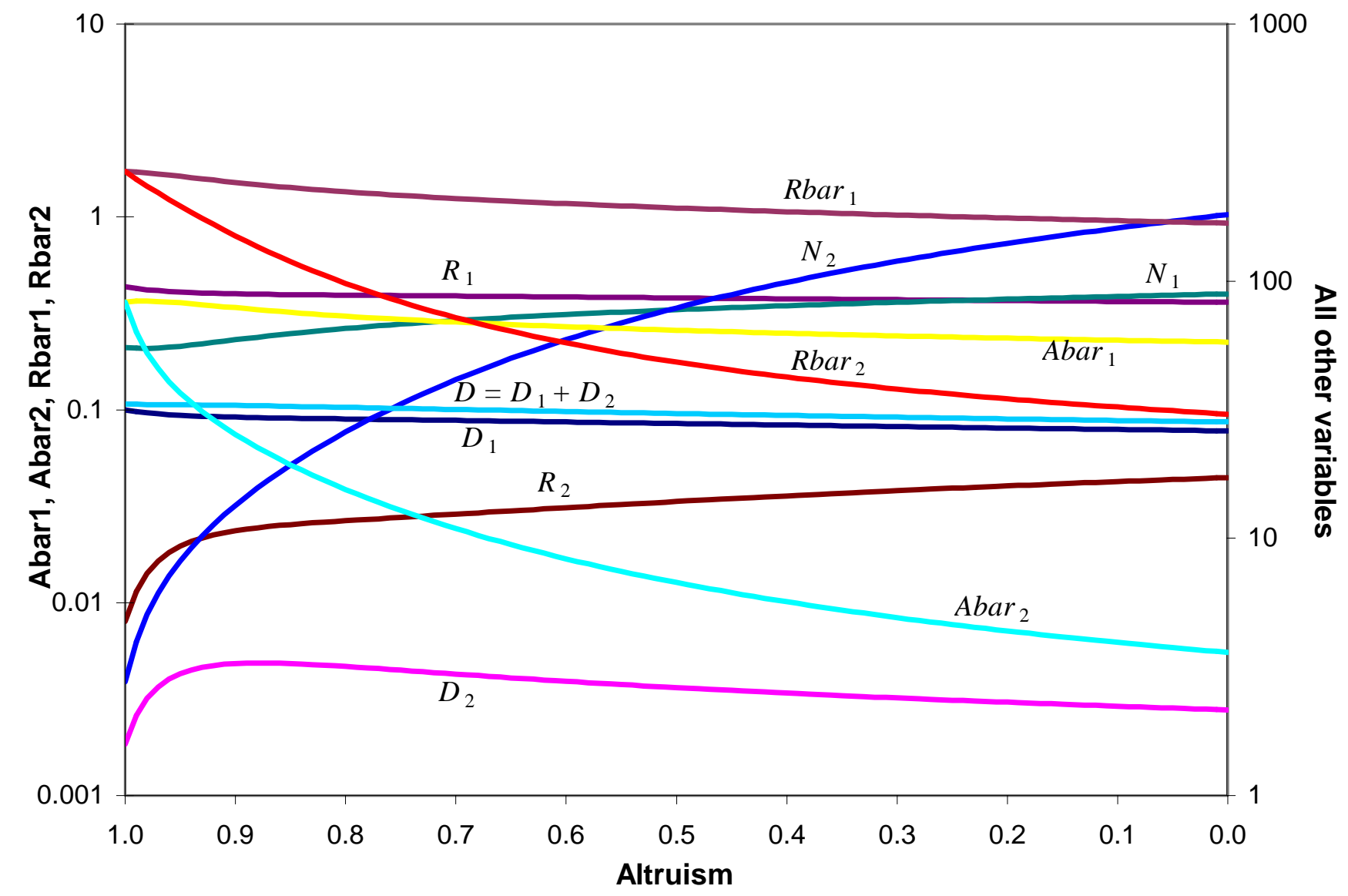

In sum, in the two-donor case, unless donors are perfectly altruistic, those with less market share proliferate more. Meanwhile, the results from the one-donor model in the previous section carry over with some modification. Simulations not reported here confirm that a change in the recipient's national-level governance quality $(G)$ or resource budget $(R)$ affect the marginal trade-offs in the donors' decisions the same way as before, so that better governance or a tighter resource budget still leads to larger projects all around.

The dependence of equilibrium project sizes on total aid (A), however, is trickier. If donor 1 , say, expands its aid spending, $A_{1}$, thus increasing $A$, the effect documented in this 
section, a greater reluctance of larger donors to proliferate, compounds the effect documented in the section before, whereby more aid leads to larger projects in order to avoid overburdening the recipient. The dependence of the equilibrium project size, $\bar{A}_{1}$, on the other donor's aid, however, is more complex. If $A_{2}$ increases while $A_{1}$ stays constant, the two effects just cited relating aid quantity and project size will sometimes counteract and sometimes reinforce each other. This is because if $A_{2}$ is much larger than $A_{1}$, further growth of $A_{2}$ gives the other donor an even more dominant role in the recipient country. And since large donors are in effect more high-minded, even greater dominance can reduce the tendency of the entire system toward competitive proliferation. At the other extreme, if $A_{2}$ is vanishingly small, to the point where the presence of the donor 2 has little effect on the sizing of donor 1's projects, then an increase in $A_{2}$ can make donor 2 into more of a competitive presence, pushing donor 1 in the direction of proliferation.

For higher numbers of donors, the situation becomes more challenging to analyze, whether with calculus or simulations. But the implications from the two-donor example seem clear. A given donor's average project size is positively related to its aid budget and the country’s governance and negatively related to the recipient's resource budget. Its relationship to total aid of the other donors is more complex, and depends on the degree of fractionalization of the other donors as a group. For example, if donor $j$ accounts for $5 \%$ of the aid to a country, its projects should be much bigger if the other $95 \%$ is accounted for by a single donor than if it is divided evenly among 100 small ones. In the latter case, there should be much more competitive proliferation, assuming donors are not perfectly altruistic, driving project sizes downward. Empirically, $A-A_{i}$ should interact with measures of fractionalization to influence $\bar{A}_{i}$. 


\section{Conclusions}

One core idea of the model developed here is that the administrative burden for the recipient associated with aid projects has both costs and benefits. Oversight can improve recipient administration of aid projects, but at the expense of diverting limited recipient resources from more directly productive use. The other key assumption is that larger projects involve proportionally less oversight burden. The conclusions that flow from the model are that projects should be larger in recipients where aid is higher in absolute terms; where recipient resources, which might be proportional to absolute GDP or tax revenue, are scarcer; and where nationallevel governance is better.

The other core idea is that while donors can compete for recipient resources, they do not do so through a market. As a result, assuming donors are not perfectly altruistic, there is a tendency in the aid system toward competitive proliferation, which reduces overall development. Coordination among donors could solve this problem, but small donors may be least interested in coordinating since they can gain from proliferation. Bringing them into a coordination regime will require what economists would call pay-offs, but which could in fact take many forms, from quiet pressure to sharing of office space. Alternatively, donors and recipients construct markets in recipient resources, perhaps through auctions. But it is hard to see how to make this practical.

Whether the assertions above about where projects "should" be bigger is best read in a normative or descriptive sense is less clear. If the assumptions about the costs and benefits of oversight activities are realistic, then the modeling results about optimal project size can be read as a guide for increasing aid effectiveness. If one makes the even stronger assumptions that donors understand these dynamics and that development results, at least from their own projects, dominate their objective functions, then the results can be read as predictions about donor behavior. Testing those predictions is separate project. 
One interesting and substantial extension to the model would be to make it dynamic, by endogenizing governance and/or recipient resources. Both could depend monotonically on development, itself the outcome of the production processes modeled here. Or donors could increase recipient resources directly, through capacity-building aid. 


\section{References}

Alesina, A., and Dollar, D. (1998), “Who Gives Foreign Aid to Whom and Why?” NBER working paper 6612, June.

Birdsall, N. (2005), “Seven Deadly Sins: Reflections on Donor Failings,” Working Paper 50, Center for Global Development, December.

Collier, P. and Dollar, D. (2002). ‘Aid Allocation and Poverty Reduction', European Economic Review, vol. 45(1): 1-26.

Kaplan, S.S. (1975), “The Distribution of Aid to Latin America: A Cross-national Aggregate Data and Time Series Analysis,” Journal of Developing Areas 10.

Knack, S., and Rahman, A. (2004), “Donor fragmentation and bureaucratic quality in aid recipients,” World Bank Policy Research Working Paper 3186, Washington, DC.

McKinley, R.D., and Little, R. (1979), “The US Aid Relationship: A Test of the Recipient Need and the Donor Interest Models,” Political Studies XXVII (2).

Morss, E.R. (1984), "Institutional Destruction Resulting from Donor and Project Proliferation in Sub-Saharan African Countries’. World Development, 12 (4): 465-70.

Mosley, P., "Models of the Aid Allocation Process: A Comment on McKinley and Little," Political Studies XXIX (2), 1981.

Moss, T., Roodman, D., and Standley, S. (2005), “The Global War on Terror and U.S. Development Assistance: USAID allocation by country, 1998-2005,” Working Paper 62, Center for Global Development, Washington, DC, July.

Roodman, D. (2006), “Aid project proliferation and absorptive capacity,” WIDER Research Paper 2006/04, Helsinki.

Schraeder, P.J., Hook, S.W., and Taylor, B. (1998), “Clarifying the Foreign Aid Puzzle: A Comparison of American, Japanese, French, and Swedish Aid Flows,” World Politics 50.

van de Walle, N., and Johnston, T. (1996), Improving Aid to Africa. Baltimore, MD: Johns Hopkins University Press for the Overseas Development Council. 


\section{Appendix 1. Comparative statics with endogenous aid and one donor}

This section demonstrates that if aid is a free parameter for optimization, along with the project count, and there is a single donor who jointly maximizes development with the recipient, then a) the optimal aid level, $A^{*}$, and optimal project count, $N^{*}$, if they exist, are directly proportional to the recipient's resource budget, $R$, thus that the optimal project size is invariant with respect to $R$; and b) the optimal aid level and project size are positively related to the recipient's governance quality, $G$.

A few identities are helpful for a formal demonstration. If we take $G$ as fixed for the moment, then $o$ is homogeneous of degree 0 in $R, A$, and $N$, as is $g$ in $A$ and $N$. This is because $o$ then depends only on $\bar{A}$ and $\bar{R}$ while $g$ depends only on $\bar{A}$. (Equations (2) define $o$ and $g$.) It follows that $D=N \cdot O \cdot g$ is homogeneous of degree 1 in these three parameters. According to Euler's Theorem,

$$
\frac{\partial D}{\partial R} R+\frac{\partial D}{\partial A} A+\frac{\partial D}{\partial N} N=D \text { and, equivalently, } \varepsilon_{R}^{D}+\varepsilon_{A}^{D}+\varepsilon_{N}^{D}=1
$$

where $\varepsilon$ means partial elasticity. One implication is that if $R, A$, and $N$, all expand by the same percentage, meaning that inputs per project hold constant while the project count grows, then so does $D$. Unsurprisingly, doubling the number of identical projects doubles development. Similarly, if $P$ represents one of the parameters $R, A$, or $N$, then $\partial D / \partial P$ is homogeneous of degree 0. Applying Euler's Theorem to $\partial D / \partial P$ yields

$$
\frac{\partial^{2} D}{\partial P \partial R} R+\frac{\partial^{2} D}{\partial P \partial A} A+\frac{\partial^{2} D}{\partial P \partial N} N=0 \text { and } \varepsilon_{R}^{\partial D / \partial P}+\varepsilon_{A}^{\partial D / \partial P}+\varepsilon_{N}^{\partial D / \partial P}=0
$$

As a final identity, note that the second partial derivatives of $D$ with respect to $R, A$, and $N$ are homogeneous of degree -1 . 
To formally examine how the optimal aid level and project size depend on the recipient resource budget, $R$, note that at an interior solution,

$$
\begin{aligned}
& \frac{\partial D}{\partial A}=0 \\
& \frac{\partial D}{\partial N}=0
\end{aligned}
$$

and the Hessian of $D$ with respect to $A$ and $N$ is negative semi-definite. We want to prove that if $A^{*}$ and $N^{*}$ are optimal at $R$, then $k A^{*}$ and $k N^{*}$ are optimal at $k R, k$ any positive number. That is, expanding the recipient's resource budget leads, in the optimum, to a proportional multiplication in the number of projects, thus no change in their size.

Since $\partial D / \partial A$ and $\partial D / \partial N$ are homogeneous of degree 0 in $R, A, N$, they remain unchanged (still 0) when all three parameters are scaled by $k$. Meanwhile, the second derivatives in the Hessian, homogeneous of degree -1 , are all multiplied by $k^{-1}$, so that the Hessian at the new point is still negative semi-definite. Thus $\left(k A^{*}, k N^{*}\right)$ also satisfies the first- and second-order conditions when the resource budget is $k R$, and is a local maximum, as desired.

Analyzing the effects on the optimum of changing recipient governance, $G$, is more involved. We begin with total differentials of equations (17) with respect to $G$ and the free parameters, $A$ and $N$ :

$$
\begin{aligned}
& \frac{\partial^{2} D}{\partial A^{2}} d A+\frac{\partial^{2} D}{\partial A \partial N} d N+\frac{\partial^{2} D}{\partial A \partial G} d G=0 \\
& \frac{\partial^{2} D}{\partial A \partial N} d A+\frac{\partial^{2} D}{\partial N^{2}} d N+\frac{\partial^{2} D}{\partial N \partial G} d N=0
\end{aligned}
$$

Dividing through by $d G$ and rearranging, 


$$
\left[\begin{array}{ll}
\frac{\partial^{2} D}{\partial A^{2}} & \frac{\partial^{2} D}{\partial A \partial N} \\
\frac{\partial^{2} D}{\partial A \partial N} & \frac{\partial^{2} D}{\partial N^{2}}
\end{array}\right]\left[\begin{array}{c}
\frac{d A^{*}}{d G} \\
\frac{d N^{*}}{d G}
\end{array}\right]=-\left[\begin{array}{c}
\frac{\partial^{2} D}{\partial A \partial G} \\
\frac{\partial^{2} D}{\partial N \partial G}
\end{array}\right]
$$

Labeling the Hessian matrix on the left $H$, and assuming without much loss in generality that it has non-zero determinant, Cramer's Rule solves the system:

$$
\left[\begin{array}{c}
\frac{d A^{*}}{d G} \\
\frac{d N^{*}}{d G}
\end{array}\right]=-\frac{1}{|H|}\left[\begin{array}{l}
\frac{\partial^{2} D}{\partial N^{2}} \frac{\partial^{2} D}{\partial A \partial G}-\frac{\partial^{2} D}{\partial A \partial N} \frac{\partial^{2} D}{\partial N \partial G} \\
\frac{\partial^{2} D}{\partial A^{2}} \frac{\partial^{2} D}{\partial N \partial G}-\frac{\partial^{2} D}{\partial A \partial N} \frac{\partial^{2} D}{\partial A \partial G}
\end{array}\right]
$$

Multiplying the top element of each column vector by $G / A^{*}$ and the bottom by $G / N^{*}$,

$$
\left[\begin{array}{c}
\frac{d A^{*}}{d G} \frac{G}{A^{*}} \\
\frac{d N^{*}}{d G} \frac{G}{N^{*}}
\end{array}\right]=-\frac{1}{|H|}\left[\begin{array}{l}
\frac{\partial^{2} D}{\partial N^{2}} \frac{\partial^{2} D}{\partial A \partial G} \frac{G}{A^{*}}-\frac{\partial^{2} D}{\partial A \partial N} \frac{\partial^{2} D}{\partial N \partial G} \frac{G}{A^{*}} \\
\frac{\partial^{2} D}{\partial A^{2}} \frac{\partial^{2} D}{\partial N \partial G} \frac{G}{N^{*}}-\frac{\partial^{2} D}{\partial A \partial N} \frac{\partial^{2} D}{\partial A \partial G} \frac{G}{N^{*}}
\end{array}\right] .
$$

Repeatedly applying the identities $\frac{\partial X}{\partial Y}=\varepsilon_{Y}^{X} \frac{Y}{X}$ and $\frac{\partial^{2} X}{\partial Y \partial Z}=\varepsilon_{Z}^{\partial X / \partial Y} \varepsilon_{Y}^{X} \frac{X}{Y Z}$,

$$
\begin{aligned}
& {\left[\begin{array}{l}
e_{G}^{A^{*}} \\
e_{G}^{N^{*}}
\end{array}\right]=-\frac{1}{|H|} \lim _{A, N \rightarrow A^{*}, N^{*}}\left[\begin{array}{l}
\varepsilon_{N}^{\partial D / \partial N} \varepsilon_{N}^{D} \frac{D}{N^{2}} \varepsilon_{A}^{\partial D / \partial G} \varepsilon_{G}^{D} \frac{D}{A G} \frac{G}{A}-\varepsilon_{A}^{\partial D / \partial N} \varepsilon_{N}^{D} \frac{D}{A N} \varepsilon_{N}^{\partial D / \partial G} \varepsilon_{G}^{D} \frac{D}{N G} \frac{G}{A} \\
\varepsilon_{A}^{\partial D / \partial A} \varepsilon_{A}^{D} \frac{D}{A^{2}} \varepsilon_{N}^{\partial D / \partial G} \varepsilon_{G}^{D} \frac{D}{N G} \frac{G}{N}-\varepsilon_{N}^{\partial D / \partial A} \varepsilon_{A}^{D} \frac{D}{A N} \varepsilon_{A}^{\partial D / \partial G} \varepsilon_{G}^{D} \frac{D}{A G} \frac{G}{N}
\end{array}\right]} \\
& =-\frac{\varepsilon_{G}^{D}}{|H|} \frac{D^{*}}{\left(A^{*} N^{*}\right)^{2}} \lim _{A, N \rightarrow A^{*}, N^{*}}\left[\begin{array}{l}
\varepsilon_{N}^{D}\left(\varepsilon_{N}^{\partial D / \partial N} \varepsilon_{A}^{\partial D / \partial G}-\varepsilon_{A}^{\partial D / \partial N} \varepsilon_{N}^{\partial D / \partial G}\right) \\
\varepsilon_{A}^{D}\left(\varepsilon_{A}^{\partial D / \partial A} \varepsilon_{N}^{\partial D / \partial G}-\varepsilon_{N}^{\partial D / \partial A} \varepsilon_{A}^{\partial D / \partial G}\right)
\end{array}\right]
\end{aligned}
$$

Since $\partial D / \partial A$ and $\partial D / \partial N$ are zero at the optimum, some of the elasticities above are zero or infinite there, which necessitates the limit operator. If the elasticities on the right-hand side were re-expressed in derivatives, $\partial D / \partial A$ and $\partial D / \partial N$ factors would arise and cancel out, eliminating the apparent singularity.

The next step is to return to the definition of $D$ in terms of $o$ and $g$ and their parameters in order to delve into the elasticities in (19). Now, 


$$
\begin{gathered}
\frac{\partial D}{\partial G}=\frac{\partial}{\partial G}\left[N \cdot o \cdot g\left((A / N)^{c-1}, G\right)\right]=N \cdot o \cdot g_{2} \\
\varepsilon_{N}^{\partial D / \partial G}=\varepsilon_{N}^{N \cdot o \cdot g_{2}}=\varepsilon_{N}^{\frac{N \cdot o \cdot g_{2}}{N \cdot o \cdot g} N \cdot o \cdot g}=\varepsilon_{N}^{g_{2} / g}-\varepsilon_{N}^{N \cdot o \cdot g}=\varepsilon_{N}^{g_{2} / g}-\varepsilon_{N}^{D} .
\end{gathered}
$$

At an interior optimum, $\varepsilon_{N}^{D}=0$, so $\varepsilon_{N}^{\partial D / \partial G}=\varepsilon_{N}^{g_{2} / g}=\varepsilon_{N}^{g_{2}}-\varepsilon_{N}^{g}$. By an identical argument,

$$
\varepsilon_{A}^{\partial D / \partial G}=\varepsilon_{A}^{g_{2}}-\varepsilon_{A}^{g} .
$$

Because $g_{2}$, like $g$, depends only on $\bar{A}$, not $A$ and $N$ separately, it too is homogeneous of degree 0 with respect to $A$ and $N$. Thus $\varepsilon_{N}^{g}+\varepsilon_{A}^{g}=0, \varepsilon_{N}^{g_{2}}+\varepsilon_{A}^{g_{2}}=0$, whence the quantities in (20) and (21) are equal and opposite. Substituting into (19),

$$
\begin{aligned}
{\left[\begin{array}{c}
e_{G}^{A^{*}} \\
e_{G}^{N^{*}}
\end{array}\right] } & =-\frac{\varepsilon_{G}^{D}}{|H|} \frac{D^{*}}{\left(A^{*} N^{*}\right)^{2}} \lim _{A, N \rightarrow A^{*}, N^{*}}\left[\begin{array}{c}
\varepsilon_{N}^{D} \varepsilon_{A}^{g_{2} / g}\left(\varepsilon_{N}^{\partial D / \partial N}+\varepsilon_{A}^{\partial D / \partial N}\right) \\
\varepsilon_{A}^{D} \varepsilon_{A}^{g_{2} / g}\left(-\varepsilon_{A}^{\partial D / \partial G}-\varepsilon_{N}^{\partial D / \partial A}\right)
\end{array}\right] \\
& =\frac{\varepsilon_{G}^{D} \varepsilon_{A}^{g_{2} / g}}{|H|} \frac{D^{*}}{\left(A^{*} N^{*}\right)^{2}} \lim _{A, N \rightarrow A^{*}, N^{*}}\left[\begin{array}{c}
\varepsilon_{N}^{D} \varepsilon_{R}^{\partial D / \partial N} \\
-\varepsilon_{A}^{D} \varepsilon_{R}^{\partial D / \partial A}
\end{array}\right] \text { (by (15)) } \\
& \left.=\frac{\varepsilon_{G}^{D} \varepsilon_{A}^{g_{2} / g}}{|H|} \frac{D^{*}}{\left(A^{*} N^{*}\right)^{2}}\left[\begin{array}{c}
\varepsilon_{R}^{D} \varepsilon_{N}^{\partial D / \partial R} \\
-\varepsilon_{R}^{D} \varepsilon_{A}^{\partial D / \partial R}
\end{array}\right] \text { (using the identity } \varepsilon_{Y}^{X} \varepsilon_{Z}^{\partial X / \partial Y}=\varepsilon_{Z}^{X} \varepsilon_{Y}^{\partial X / \partial Z}\right) \\
& =\frac{\varepsilon_{G}^{D} \varepsilon_{R}^{D} \varepsilon_{A}^{g_{2} / g}}{|H|} \frac{D^{*}}{\left(A^{*} N^{*}\right)^{2}}\left[\begin{array}{c}
\varepsilon_{N}^{\partial D / \partial R} \\
\varepsilon_{N}^{\partial D / \partial R}+\varepsilon_{R}^{\partial D / \partial R}
\end{array}\right] \text { (by (15)). }
\end{aligned}
$$

To demonstrate that the optimal aid level and project size rise with $G$, i.e., that $e_{G}^{A^{*}}>0$ and $e_{G}^{N^{*}}<e_{A}^{N^{*}}$ (these elements constitute the left-hand side) we need to show that all the terms outside the right-hand-side column vector in the last expression are positive and that, inside the vector, $\varepsilon_{N}^{\partial D / \partial R}>0$ and $\varepsilon_{N}^{\partial D / \partial R}+\varepsilon_{R}^{\partial D / \partial R}<\varepsilon_{N}^{\partial D / \partial R}$. The positivity of $\varepsilon_{R}^{D}$ and $\varepsilon_{G}^{D}$ follow directly from (2) and (5). $|H|>0$ because $H$ is negative definite with even dimension (its two eigenvalues are negative and their product is $|H|$ ). As for $\varepsilon_{A}^{g_{2} / g}\left(=\varepsilon_{A}^{g_{2}}-\varepsilon_{A}^{g}\right)$, in general, if $A$ increases, then the first argument of $g$, the ratio of monitoring cost to project size, decreases, and so does $g$. Thus 
$\varepsilon_{A}^{g}<0$. And since $g_{12}<0, \varepsilon_{A}^{g_{2}}>0$. So $\varepsilon_{A}^{g_{2} / g}$ is positive. Thus all the outside terms on the right side of (22) are positive, as needed.

It remains to analyze $\varepsilon_{N}^{\partial D / \partial R}$ and $\varepsilon_{R}^{\partial D / \partial R}$, the two terms in the right-hand-side vector. The elasticity $\varepsilon_{R}^{\partial D / \partial R}$ must be negative because it equals $\frac{\partial^{2} D}{\partial R^{2}} \frac{1}{\partial D / \partial R} R$; and $\frac{\partial D}{\partial R}>0$, and $\frac{\partial^{2} D}{\partial R^{2}}<0$ at the solution, a maximum of $D$ with respect $R$. This confirms that the bottom term of the right-handside vectors is less than the top term. We study $\varepsilon_{N}^{\partial D / \partial R}$ much the way we did $\varepsilon_{A}^{\partial D / \partial G}$, in (20):

$$
\begin{aligned}
\frac{\partial D}{\partial R}= & \frac{\partial}{\partial R}\left[N \cdot o\left(A / N, R / N-(A / N)^{c}\right) \cdot g\right]=o_{2} \cdot g \\
\varepsilon_{N}^{\partial D / \partial R} & =\varepsilon_{N}^{o_{2} \cdot g}=\varepsilon_{N}^{\frac{o_{2} \cdot g}{N \cdot o \cdot g} N \cdot o \cdot g}=\varepsilon_{N}^{o_{2} / N \cdot o}-\varepsilon_{N}^{N \cdot o \cdot g}=\varepsilon_{N}^{o_{2} / o}-1-\varepsilon_{N}^{D} \\
& =\varepsilon_{N}^{o_{2} / o}-1 \text { at the solution, a maximum with respect to } N .
\end{aligned}
$$

Since $o$ is homogeneous of degree $d$ in its two arguments $\left(\bar{A}\right.$ and $\left.\bar{R}-\bar{A}^{c}\right), o_{2}$ is homogeneous of degree $d-1$, and $o_{2} / o$, treated as a single function, is homogeneous of degree -1 : its elasticities with respect to its arguments sum to -1 . Using the chain rule for elasticities,

$$
\begin{aligned}
\varepsilon_{N}^{o_{2} / o} & =\varepsilon_{1}^{o_{2} / o} \varepsilon_{N}^{\bar{A}}+\varepsilon_{2}^{o_{2} / o} \varepsilon_{N}^{\bar{A}-\bar{R}^{c}}=\left(\varepsilon_{1}^{o_{2} / o}+\varepsilon_{2}^{o_{2} / o}\right) \varepsilon_{N}^{\bar{A}}+\varepsilon_{2}^{o_{2} / o}\left(\varepsilon_{N}^{\bar{R}-\bar{A}^{c}}-\varepsilon_{N}^{\bar{A}}\right) \\
& =(-1) \varepsilon_{N}^{\bar{A}}+\varepsilon_{2}^{o_{2} / o}\left(\varepsilon_{N}^{\bar{R}-\bar{A}^{c}}-\varepsilon_{N}^{\bar{A}}\right) \\
& =1-\varepsilon_{2}^{o_{2} / o}(1-c) \frac{\bar{A}^{c}}{\bar{R}-\bar{A}^{c}}
\end{aligned}
$$

The last step here uses $\varepsilon_{N}^{\bar{A}}=-1$ and $\varepsilon_{N}^{\bar{R}-\bar{A}^{c}}=-\frac{\bar{R}-c \bar{A}^{c}}{\bar{R}-\bar{A}^{c}}$, which can be derived using the sum rule for elasticities, namely $\varepsilon_{Z}^{X+Y}=\frac{X \varepsilon_{Z}^{X}+Y \varepsilon_{Z}^{Y}}{X+Y}$. Substituting into (23),

$$
\varepsilon_{N}^{\partial D / \partial R}=\varepsilon_{N}^{o_{2} / o}-1=-\varepsilon_{2}^{o_{2} / o}(1-c) \frac{\bar{A}^{c}}{\bar{R}-\bar{A}^{c}}=\left(\varepsilon_{2}^{o}-\varepsilon_{2}^{o_{2}}\right)(1-c) \frac{\bar{A}^{c}}{\bar{R}-\bar{A}^{c}}
$$

By (4), $\varepsilon_{2}^{o} \geq 0$ and $\varepsilon_{2}^{o_{2}} \leq 0$. Moreover, $c<1$ and, by at an interior solution, the excess of the 
recipient's resource input over monitoring cost, $\bar{R}-\bar{A}^{c}$, is positive. As a result, $\varepsilon_{N}^{\partial D / \partial R}$ is positive, as claimed. Better-governed countries should get bigger projects as well as more aid. 


\section{Appendix 2. Comparative statics with exogenous aid and one donor}

This section demonstrates that if the donor's aid budget is given and donor and recipient jointly

maximize development, then projects will be larger where there is more aid, where the recipient's resource budget is tighter in absolute terms, and larger where the recipient's governance is better-all else equal.

For each parameter of interest, $P$ (meaning $A, R$, or $G$ ), the first-order condition $\partial D / \partial N=0$ defines an isoquant in $P-N$ space because for each value of $P$ there is an optimal project count, $N$, called $N^{*}$. The slope of the isoquant is derived as:

$$
\frac{\partial N^{*}}{\partial P}=-\frac{\frac{\partial}{\partial P}\left(\frac{\partial D}{\partial N}\right)}{\frac{\partial}{\partial N}\left(\frac{\partial D}{\partial N}\right)}=-\frac{\frac{\partial^{2} D}{\partial P \partial N}}{\frac{\partial^{2} D}{\partial N^{2}}} .
$$

This can be translated into elasticities in two useful ways:

$$
\begin{gathered}
\varepsilon_{P}^{N^{*}}=-\lim _{N \rightarrow N^{*}} \frac{P \frac{\partial D}{\partial P} \varepsilon_{N}^{\partial D / \partial P}}{N \frac{\partial D}{\partial N} \varepsilon_{N}^{\partial D / \partial N}} \\
\varepsilon_{P}^{N^{*}}=-\lim _{N \rightarrow N^{*}} \frac{\varepsilon_{P}^{\partial D / \partial N}}{\varepsilon_{N}^{\partial D / \partial N}} .
\end{gathered}
$$

If the elasticities on the right-hand sides are expanded using the identity $\varepsilon_{B}^{A}=\frac{\partial A}{\partial B} \frac{B}{A}$, all the $\frac{\partial D}{\partial N}$ factors cancel out. But since they are present and zero at $N=N^{*}$, the fractions are technically indeterminate there, which necessitates the limit operators. By (12), the denominator of the grand fraction in all three versions is negative. For two parameters of interest $-P=G$ or $R$-both $P$ and $\partial D / \partial P$ are positive everywhere. So for these two, (28) shows that the task of determining the sign of $\varepsilon_{P}^{N^{*}}$ boils down to determining the sign of $\varepsilon_{N}^{\partial D / \partial P}$. We will perform the task for those two 
first, then move to the third parameter, $A$.

For $P=G$, Appendix 1 shows that $\varepsilon_{N}^{\partial D / \partial G}=\varepsilon_{N}^{g_{2} / g}$ (equation (21)), and that this is negative at an optimum with respect to $N$. According to (28), so is $\varepsilon_{G}^{N^{*}}$ : better national-level governance favors fewer projects, thus, holding total aid constant, larger projects.

As for the dependence of optimal project count on the recipient resource budget, $R$, Appendix 1 also demonstrates that

$$
\varepsilon_{N}^{\partial D / \partial R}=\left(\varepsilon_{2}^{o}-\varepsilon_{2}^{o_{2}}\right)(1-c) \frac{\bar{A}^{c}}{\bar{R}-\bar{A}^{c}},
$$

and that this is positive, which by (28) means $\varepsilon_{R}^{N^{*}}$ is too. In words, the larger the recipient's budget, the higher the optimal project count, and the smaller the optimal project size, all else equal.

To determine, $\varepsilon_{A}^{N^{*}}$, the dependence of the optimum project count on the final parameter, $A$, we take advantage of Euler's Theorem again. Setting $P=A$ in (16), solving for $\varepsilon_{A}^{\partial D / \partial N}$ and substituting into (29),

$$
\varepsilon_{A}^{N^{*}}=1+\lim _{N \rightarrow N^{*}} \frac{\varepsilon_{R}^{\partial D / \partial N}}{\varepsilon_{N}^{\partial D / \partial N}}
$$

We have seen that $\varepsilon_{N}^{\partial D / \partial N}<0$ and $\varepsilon_{R}^{\partial D / \partial N}>0$ at $N^{*}$, thus near it. Ergo $\varepsilon_{A}^{N^{*}}<1$. More aid makes fewer projects optimal. 


\section{Appendix 3. Comparative statics with exogenous aid and two donors}

This appendix derives two results used in section 0 for the two-donor case: that at the development optimum, each donor, unless perfectly altruistic about the success of the other's projects, has an incentive to proliferate, increasing its project count in order to lure recipient resources away from the other donor's projects in a way that is detrimental to overall development; and that the temptation is greatest, in a certain sense, for small donors.

We start with the problems defined in (13) and (14), with two donors. Taking the donors choices as given for the moment and focusing on the recipient's problem, a Lagrangian argument shows, as usual, that the recipient's solution exhausts its budget and equalizes the marginal product of its resource across donors:

$$
\begin{aligned}
& R_{1}+R_{2}=R \\
& \frac{\partial D_{1}}{\partial R_{1}}=\frac{\partial D_{2}}{\partial R_{2}} .
\end{aligned}
$$

Now, to describe how the recipient responds to a change in a donor's behavior, we differentiate the above with respect to $N_{1}$ while fixing $N_{2}$ :

$$
\begin{aligned}
& \frac{d R_{1}^{*}}{d N_{1}}=-\frac{d R_{2}^{*}}{d N_{1}} \\
& \frac{\partial^{2} D_{1}}{\partial N_{1} \partial R_{1}}+\frac{\partial^{2} D_{1}}{\partial R_{1}^{2}} \frac{d R_{1}^{*}}{d N_{1}}=\frac{\partial^{2} D_{2}}{\partial R_{2}^{2}} \frac{d R_{2}^{*}}{d N_{1}}
\end{aligned}
$$

(using the chain rule in the second equation). Substituting the first equation into the second and rearranging gives a formula for how the recipient reallocates its resource in response to donor 1 dividing its aid budget more finely: 


$$
\frac{d R_{1}^{*}}{d N_{1}}=-\frac{\frac{\partial^{2} D_{1}}{\partial N_{1} \partial R_{1}}}{\frac{\partial^{2} D_{1}}{\partial R_{1}^{2}}+\frac{\partial^{2} D_{2}}{\partial R_{2}^{2}}}
$$

Since $D_{2}$ can be viewed as a function of $R_{1}$ via the constraint $R_{2}=R-R_{1}$, we can write $\partial D_{2} / \partial R_{1}=-\partial D_{2} / \partial R_{2}$ and $\partial^{2} D_{2} / \partial R_{1}^{2}=\partial^{2} D_{2} / \partial R_{2}^{2}$. Substituting into the above,

$$
\frac{d R_{1}^{*}}{d N_{1}}=-\frac{\frac{\partial^{2} D_{1}}{\partial N_{1} \partial R_{1}}}{\frac{\partial^{2} D_{1}}{\partial R_{1}^{2}}+\left(\left.\frac{\partial^{2} D_{2}}{\partial R_{1}^{2}}\right|_{R_{1}+R_{2}+R}\right)}=-\frac{\frac{\partial^{2} D_{1}}{\partial N_{1} \partial R_{1}}}{\left.\frac{\partial^{2} D}{\partial R_{1}^{2}}\right|_{R_{1}+R_{2}+R}} .
$$

The denominator of the final expression is the second derivative of total development with respect to the recipient's choice of $R_{1}$; it is negative since at the development optimum we are analyzing, the recipient achieves a maximum of $D$ with respect to $R_{1}$. The numerator is positive by arguments identical to those used in the Appendix 1 to show that $\varepsilon_{N}^{\partial D / \partial R}>0$ (23), meaning that dividing the aid budget more finely increases the marginal product of the recipient's resource. The overall result is that $d R_{1}^{*} / d N_{1}$ is positive. So when a donor raises its project count while holding total aid constant, it attracts the recipient's resource away from the other donor. A corollary is that the development optimum is not a Nash equilibrium if donors factor the recipient's behavior into their choices, unless the donors are perfectly altruistic. To see this, note that the marginal effect on output from donor 1's projects if it proliferates is, by the chain rule,

$$
\frac{d D_{1}^{*}}{d N_{1}}=\frac{\partial D_{1}}{\partial N_{1}}+\frac{\partial D_{1}}{\partial R_{1}} \frac{d R_{1}^{*}}{d N_{1}}
$$

The effect on donor 1's utility is 


$$
\frac{d D_{1}^{*}}{d N_{1}}+\alpha_{1} \frac{d D_{2}^{*}}{d N_{1}}=\frac{\partial D_{1}}{\partial N_{1}}+\frac{\partial D_{1}}{\partial R_{1}} \frac{d R_{1}^{*}}{d N_{1}}+\alpha_{1}\left(\frac{\partial D_{2}}{\partial N_{1}}+\frac{\partial D_{2}}{\partial R_{2}} \frac{d R_{2}^{*}}{d N_{1}}\right)
$$

Since $\partial D_{2} / \partial N_{1}=0\left(D_{2}\right.$ does not depend on $\left.N_{1}\right)$, since the recipient resource constraint forces $d R_{2}^{*} / d N_{1}=-d R_{1}^{*} / d N_{1}$, and since the recipient's optimizing behavior assures that $\partial D_{1} / \partial R_{1}=\partial D_{2} / \partial R_{2}$, the above can be expressed as

$$
\frac{d D_{1}^{*}}{d N_{1}}+\alpha_{1} \frac{d D_{2}^{*}}{d N_{1}}=\frac{\partial D_{1}}{\partial N_{1}}+\left(1-\alpha_{1}\right) \frac{\partial D_{1}}{\partial R_{1}} \frac{d R_{1}^{*}}{d N_{1}}
$$

At the development optimum, the first term on the right, the direct impact on donor 1's projects of proliferations, is zero. The second term, the benefit of attracting more recipient resources, is positive by (5) and (31) unless donor 1 is perfectly altruistic $\left(\alpha_{1}=1\right)$. So increasing the project count while holding aid constant serves the imperfectly altruistic donor. But of course it moves the recipient away from the development optimum, for a net loss in total development.

In fact, by the Envelope Theorem, since the recipient chooses $R_{1}$ to maximize $D$, the drop in development is

$$
\frac{d D^{*}}{d N_{1}}=\frac{\partial D}{\partial N_{1}}=\frac{\partial D_{1}}{\partial N_{1}}
$$

Right at the development optimum, this is zero. But as donor 1 moves away from the optimum, in the direction of larger projects, $\partial D_{1} / \partial N_{1}$ goes negative since $D$ is concave there with respect to $N_{1}$. Beneath the surface of this equation, the indirect effect on total development of increasing $N_{1}$, via the recipient's resource shift, is zero because the recipient is continuously operating at a margin where the development benefit of its resources is equated across donors. As a result, the direct (and negative) effect on total development of donor 1's proliferation constitutes the overall impact on development. 
The essential point here is that at and near the development optimum Donor 1 experiences little harm from proliferating, for this is the region where the derivative of $D_{1}$ with respect to $N_{1}$ vanishes. Yet increasing its project count does bring a gain by attracting recipient resources away from other donors. And because there is no competitive market in these resources, Donor 1 does not pay for them. An externality results.

The temptation to proliferation at the development optimum is greatest for smaller donors. This is most easily demonstrated for perfectly selfish donors. In this case, using the Chain Rule and adapting (35), for some donor $j$,

$$
\begin{aligned}
\frac{d D_{j}^{*}}{d N_{j}} & =\frac{\partial D_{j}}{\partial N_{j}}+\frac{\partial D_{j}}{\partial R_{j}} \frac{d R_{j}^{*}}{d N_{j}}=\frac{\partial D_{j}}{\partial N_{j}}-\frac{\partial D_{j}}{\partial R_{j}} \frac{\frac{\partial^{2} D_{j}}{\partial N_{j} \partial R_{j}}}{\frac{\partial^{2} D_{1}}{\partial R_{1}^{2}}+\frac{\partial^{2} D_{2}}{\partial R_{2}^{2}}} . \\
& =-\frac{\partial D_{j}}{\partial R_{j}} \frac{\frac{\partial^{2} D_{j}}{\partial N_{j} \partial R_{j}}}{\frac{\partial^{2} D_{1}}{\partial R_{1}^{2}}+\frac{\partial^{2} D_{2}}{\partial R_{2}^{2}}} \text { (since } \frac{\partial D_{j}}{\partial N_{j}}=0 \text { at the development optimum). }
\end{aligned}
$$

This is the marginal relationship of a selfish donor's utility to its project count. Notice that the denominator is the same for both donors. Meanwhile, the other terms on the right side together are

$$
\begin{aligned}
\frac{\partial D_{j}}{\partial R_{j}} \frac{\partial^{2} D_{j}}{\partial N_{j} \partial R_{j}} & =\frac{1}{N_{j}}\left(\frac{\partial D_{j}}{\partial R_{j}}\right)^{2} \varepsilon_{N_{j}}^{\partial D_{j} / \partial R_{j}} \\
& =\frac{1}{N_{j}}\left(o_{2} \cdot g\right)^{2} \varepsilon_{N_{j}}^{o_{2} \cdot g}
\end{aligned}
$$

At the development optimum, the donors’ projects are identical and differ only in 
number. The terms $O_{2} \cdot g$ and $\varepsilon_{N_{j}}^{o_{2} \cdot g}$ are therefore the same for both donors. Only the $1 / N_{j}$ factor above can differ between the donors. Putting all this together,

$$
N_{1} \frac{d D_{1}^{*}}{d N_{1}}=N_{2} \frac{d D_{2}^{*}}{d N_{2}} .
$$

At the development optimum, all projects are identical, so we can write $D_{j}=N_{j} \cdot o \cdot g$.

Multiplying both sides of (36) by $o \cdot g=D_{1} / N_{1}=D_{2} / N_{2}$ leads to $N_{1} e_{N_{1}}^{D_{1}^{*}}=N_{2} e_{N_{2}}^{D_{2}^{*}}$. At the development optimum, the elasticity of a donor's utility with respect to its project count is inversely related to that project count, which is itself directly proportional to donor's total aid. In this sense, smaller donors face proportionally more incentive to proliferate. 